\title{
QoS-Aware Resource Allocation for Network Virtualization in an Integrated Train Ground Communication System
}

\author{
Li Zhu $\mathbb{D}^{1},{ }^{1}$ Fei Wang, ${ }^{2}$ and Hongli Zhao ${ }^{3}$ \\ ${ }^{1}$ State Key Laboratory of Rail Traffic Control and Safety, Beijing Jiaotong University, Beijing, China \\ ${ }^{2}$ Signal and Communication Research Institute, China Academy of Railway Sciences, Beijing, China \\ ${ }^{3}$ National Engineering Research Center of Rail Transportation Operation and Control System, Beijing Jiaotong University, \\ Beijing, China \\ Correspondence should be addressed to Li Zhu; zhulibjtu@gmail.com
}

Received 28 October 2017; Revised 26 January 2018; Accepted 11 February 2018; Published 3 May 2018

Academic Editor: Daniele Pinchera

Copyright (C) $2018 \mathrm{Li}$ Zhu et al. This is an open access article distributed under the Creative Commons Attribution License, which permits unrestricted use, distribution, and reproduction in any medium, provided the original work is properly cited.

Urban rail transit plays an increasingly important role in urbanization processes. Communications-Based Train Control (CBTC) Systems, Passenger Information Systems (PIS), and Closed Circuit Television (CCTV) are key applications of urban rail transit to ensure its normal operation. In existing urban rail transit systems, different applications are deployed with independent train ground communication systems. When the train ground communication systems are built repeatedly, limited wireless spectrum will be wasted, and the maintenance work will also become complicated. In this paper, we design a network virtualization based integrated train ground communication system, in which all the applications in urban rail transit can share the same physical infrastructure. In order to better satisfy the Quality of Service (QoS) requirement of each application, this paper proposes a virtual resource allocation algorithm based on QoS guarantee, base station load balance, and application station fairness. Moreover, with the latest achievement of distributed convex optimization, we exploit a novel distributed optimization method based on alternating direction method of multipliers (ADMM) to solve the virtual resource allocation problem. Extensive simulation results indicate that the QoS of the designed integrated train ground communication system can be improved significantly using the proposed algorithm.

\section{Introduction}

With the city expansion and urban population explosion, the traditional road traffic facilities cannot satisfy the demand of modern society. Energetically developing urban rail transit system and improving the speed and capacity of rail transit have become desirable all over the world. Studies of urban rail transit have become a research focus among engineers and researchers all over the world.

The train ground communication is a key technology to ensure the normal operation of urban rail transit [1]. Most of the urban rail transit applications, such as CommunicationsBased Train Control (CBTC) Systems [2], Passenger Information Systems (PIS), and the Closed Circuit Television (CCTV), need train ground communication systems. In existing urban rail transit systems, CBTC, PIS, and CCTV adopt WLAN that use unlicensed spectrum as their train ground communication technology [3]. The construction and management work of train ground communication systems for each application are independent in existing urban rail transit systems. It is a huge waste of limited wireless spectrum and other social resources to invest and build new communication infrastructures for each application. Maintaining these infrastructures will also become a great burden. In order to ensure the safety of urban rail transit operation, integrating all these communication system into a whole is quite desirable for urban rail transit systems.

The major opportunities and challenges in train ground communication systems are summarized in [4]. Lots of researchers have studied issues related to urban rail transit train ground communication recently. Literature [5] aims to present a comprehensive tutorial, as well as a survey of the state-of-the-art of CBTC and the role of radio communication in it. A summary of the evolution of the communication 
technologies used for modern railway signalling, best practices in the design of a CBTC radio network, and the measures to optimize its availability are discussed as well. In [6], a MIMO-assisted handoff (MAHO) scheme for CBTC systems is proposed to reduce transmission and handoff delay. In [7], the Markov model of redundant and nonredundant CBTC train ground communication system structure is established to analyze the system reliability and availability. The effect of different system redundancy and the relationship between the availability of CBTC train ground communication system and the speed of train are also discussed. Channel modeling in CBTC train ground systems is intensively studied in [8, 9]. Combining Artificial Intelligence- (AI-) based decisionmaking and learning algorithms, Amanna et al. [10] present a railroad-specific cognitive radio (rail-CR) with softwaredefined radio (SDR). Based on periodical signal quality changes, the authors of [11] propose a scheduling and resource allocation mechanism to maximize the transmission rate for LTE based train ground communication system. For the handoff problem in train ground communication system, a seamless handoff scheme based on a dual-layer and duallink system architecture is proposed in [12] to reduce communication interruption time. In our previous work, crosslayer handoff designs have been studied extensively in [13] for WLANs based CBTC train ground communication systems.

These above works study urban rail transit train ground communication system performance and analyze the influence of rail transit environment on system performance. However, most of the works only focus on independent applications. Few studies take all the train ground applications into consideration. Our previous works test an LTE based integrated train ground communication system performance [14-16]. We also study the handoff design in existing integrated train ground communication systems [3]. However, the problem of improper system wireless spectrum allocation is largely ignored in these works.

In the paper, we design a network virtualization based integrated train ground communication system for urban rail transit systems. With a variety of applications, the designed system can be updated from the existing system. This kind of design not only reduces construction and operational costs but also improves the spectrum utilization efficiency. In order to better meet the QoS requirement of applications in the designed system using wireless network virtualization technology [17], this paper proposes a virtual resource allocation algorithm based on QoS guarantee, base station (BS) load balance, and application station fairness. Meanwhile, we define a QoS satisfaction level (QoSL) parameter to reflect the application satisfaction. The final optimization goal is to ensure CBTC application reliability and maximize QoS satisfaction of all the application stations.

In addition, with the further development of distributed convex optimization, we develop a distributed wireless virtual resource allocation algorithm based on alternating direction method of multipliers (ADMM) [18] to solve the virtual resource allocation problem. Simulation results indicate that the QoS of the designed integrated train ground communication system can be remarkably improved with the proposed method.
The rest of the paper is organized as follows. In Section 2, the integrated train ground communication system architecture is introduced. Section 3 describes the system model and problem formulation. The virtual resource allocation problem transformation and solution using ADMM are discussed in Sections 4 and 5, respectively. Simulation results are given in Section 6. Finally, the conclusion is given in Section 7.

\section{The Designed Integrated Train Ground Communication System Architecture}

In this section, we first introduce the QoS requirement of different applications in train ground communication and then present the basic structure of the designed integrated train ground communication system. Next, we study how each of the virtualization characteristics are conducted in a physical BS. Finally, we depict the use of network virtualization in the designed system.

2.1. Applications in Urban Rail Transit Systems. Nowadays, there are mainly three applications in urban rail transit systems. They are Communication Based Train Control (CBTC) systems, Closed Circuit Television (CCTV), and Passenger Information Systems (PIS).

As shown in Figure 1, in CBTC systems, continuous bidirectional wireless communications between the ground base station (BS) and each onboard application station are used instead of the traditional track circuit based train control system. Trains will get the state of the front train and other obstacles from the Zone Controller (ZC). It will compute a braking curve, so as to stop at a proper position. Theoretically, the distance between two trains can be just a few meters, if both trains can get the real time position of the front train and both trains have the same speed and braking capability.

However, as explained in [19], when the train behind does not get the real time position of the front train due to train ground communication delay, it will trigger the brake to stop before entering a danger zone. This process will have a significant negative impact on CBTC system performance. Therefore, the most important QoS measure of train ground communication system is transmission delay. Typical values of required transmission delay and other suggested QoS measures in CBTC system are illustrated in Table 1.

In urban rail transit system, the other two crucial applications are PIS and CCTV. Taking advantages of advanced communication and multimedia techniques, diverse multimedia information such as weather forecast, train arriving time, and advertisement will be provided to passengers on trains and in stations through PIS. CCTV is a crucial additive means to guarantee train secure operation. By using CCTV, the urban rail control center can monitor the train carriage, station, and other essential zones through continuous train ground video transmission. For the PIS and CCTV application, throughput and jitter delay are the direct performance measure, since the high quality video needs higher throughput and less jitter delay.

The suggested values of transmission data rate and other suggested QoS measures in PIS and CCTV are illustrated in 


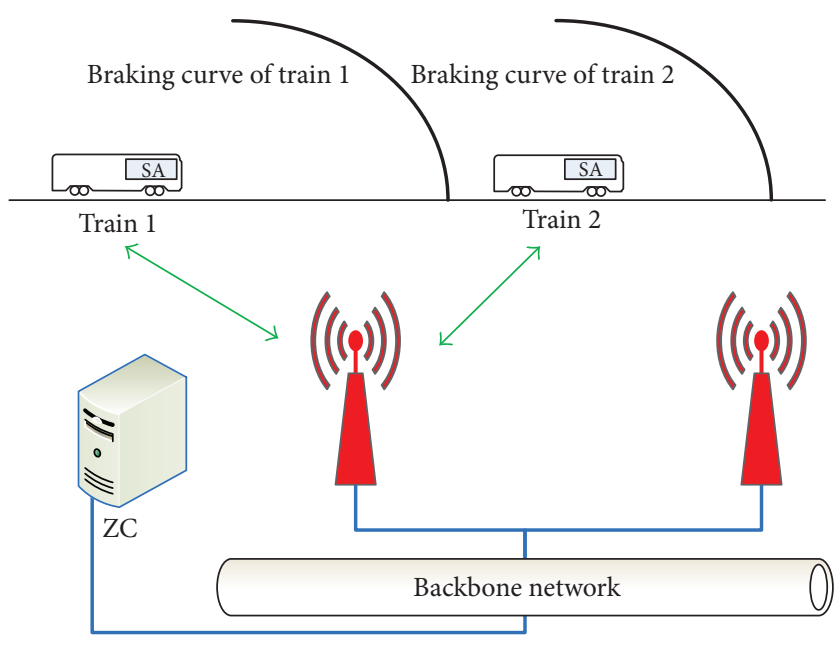

FIgUre 1: A typical CBTC system.

TABLE 1: The QoS requirement of different applications in urban rail transit systems.

\begin{tabular}{lcccccc}
\hline Number & Application & Throughput & Packet loss rate & Transmission delay & Jitter delay & Reliability \\
\hline 1 & CBTC & $100 \mathrm{kbps}$ & Less than 0.005 & $150 \mathrm{~ms}$ & None & High \\
2 & CCTV & $1 \mathrm{Mbps}$ & None & $500 \mathrm{~ms}$ & $30 \mathrm{~ms}$ & Intermediate \\
3 & PIS & $4 \mathrm{Mbps}$ & None & $500 \mathrm{~ms}$ & $30 \mathrm{~ms}$ & Intermediate \\
\hline
\end{tabular}

Table 1 . We get this table by consulting several urban rail operation corporations in China. The data in Table 1 is not absolute performance requirement standard. As a matter of fact, urban rail operation corporations (especially corporations in different cities) have different communication QoS requirement for train ground applications. The performance requirement in this table is obtained by synthesizing various data from different corporations. We need to point out that the proposed optimization algorithm in our designed integrated train ground communication system is not dependent on the data in Table 1. Once we get more authoritative performance requirement parameters, they can be used in our optimization model and more accurate simulation results can be obtained.

The construction and management works of train ground communication system for each application are independent in existing urban rail transit system. It is a huge waste of limited wireless spectrum and other social resources to invest and build new communication infrastructures for each application. Recently, engineers try to design a system that combines all the applications together. The system architecture is shown in Figure 2. In order to improve CBTC system reliability, two independent ground infrastructures are used. There are two CBTC application stations on the train, which are installed on its nose and tail, and they are connected to different ground infrastructures. Two independent train ground infrastructures are allocated with constant spectrum. The PIS and CCTV application stations only connect to one of the ground infrastructures and share wireless spectrum with CBTC systems.

One disadvantage of the above system is the improper spectrum resource allocation scheme. The designed system using two independent ground infrastructures guarantees the CBTC system reliability. However, the spectrum allocated to urban rail transit systems is limited, and all the channels used by different applications share the same spectrum. Channels needed by different applications are dynamically changing, and allocating constant channels to different applications will waste limited spectrum resource.

In order to better satisfy the QoS requirement of different applications, we design an integrated train ground communication system for urban rail transit system using wireless network virtualization techniques, which will be introduced in the next subsections.

\subsection{Architecture of the Designed Integrated Train Ground} Communication System. The designed system architecture is shown in Figure 3. Different from the existing system, in our designed system, the two infrastructures can be connected by PIS and CCTV application stations as well as both the two CBTC application stations.

The proposed integrated train ground communication system architecture is shown in Figure 4. For a certain railway line, it is assumed that there is only one physical infrastructure provider $(\mathrm{PiP})$, which provides three different network services to the train with three different application stations. According to the general wireless network virtualization definition, the proposed architecture can be divided into two separate layers: the control and management layer (CML) and the virtualization layer (VL).

The main responsibility of CML is resource management. The main functions of CML are realized by several virtual network controllers and a hypervisor. Every virtual network has 


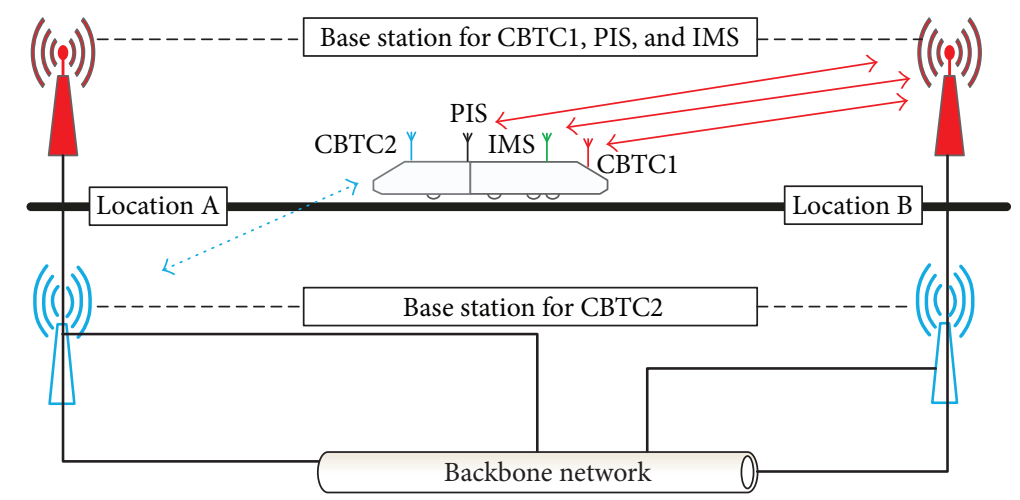

FIGURE 2: Existing integrated train ground communication systems.

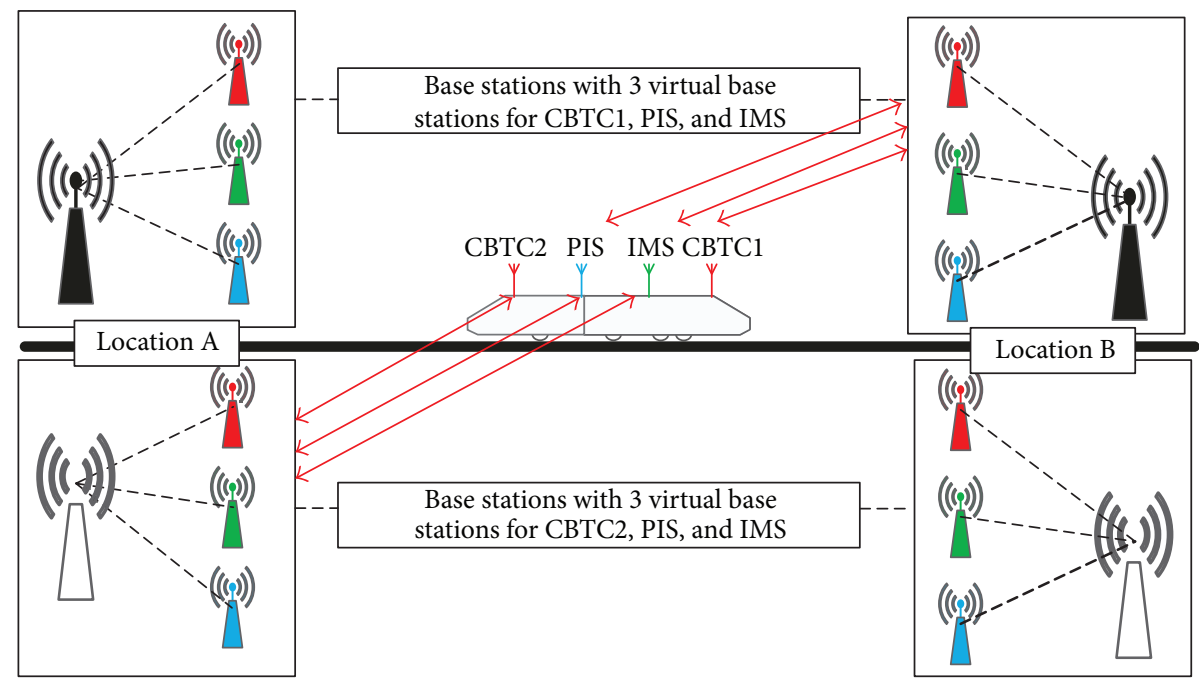

FIgURE 3: The proposed integrated train ground communication system.

its own network controller, which is responsible for scheduling application stations, determining their QoS requirement, and informing the hypervisor of them. The hypervisor can flexibly allocate the virtual resources to virtual networks under different circumstances according to the feedback information (transmit power, e.g., and available spectrum) and different QoS requirements. The whole network has one hypervisor. By using wireless network virtualization, each application station could be served via the same PiPs and different spectrum resources.

The VL is accountable for the abstraction, programmability, and isolation of physical resources in a certain physical base station (BS). Using various VL functions, the PiP will be able to broadcast beacons for virtual BSs of various applications. In addition, each of the virtual networks should have independent control of settings in their virtual BSs. They can set different attributes for virtual BSs, such as various security policies, broadcast domains, and IP settings. Furthermore, the virtual BSs can be isolated by different wireless spectrum.

The VL also provides the CML with the interfaces needed to control virtualized resources (spectrums, transmission power, etc.). With VL, both the PiP and the wireless resources are virtualized and shared by various virtual networks.

The virtual resource allocation is one key issue in the above system. Physical and wireless virtual resources should be dynamically allocated to the CBTC, PIS, and CCTV according to their requirement. If the virtual resource allocation scheme is not carefully designed, the normal CBTC system function will not be ensured. The video transmission quality of PIS and CCTV will degrade. This will have a significant negative impact on urban rail transit system. To this point, we will study virtual resource allocation schemes in the following sections.

\section{System Model and Problem Formulation}

In the designed system, we define $\mathcal{N}$ as the base station (BS) sets, $\mathcal{N}=\{1,2, \ldots, N\}$. The integrated system is virtualized into multiple virtual BSs (VBSs) for different services. The system has a set $\mathscr{K}$ of VBSs, $\mathscr{K}=\{1,2, \ldots, K\}$. For each VBS $k, k \in \mathscr{K}, \mathscr{V}_{k}$ is the set of application stations of VBS $k$, and $v_{k}$ is one of application stations served by VBS $k$, $v_{k} \in \mathscr{V}_{k}$. In the integrated system, a wireless channel is a 


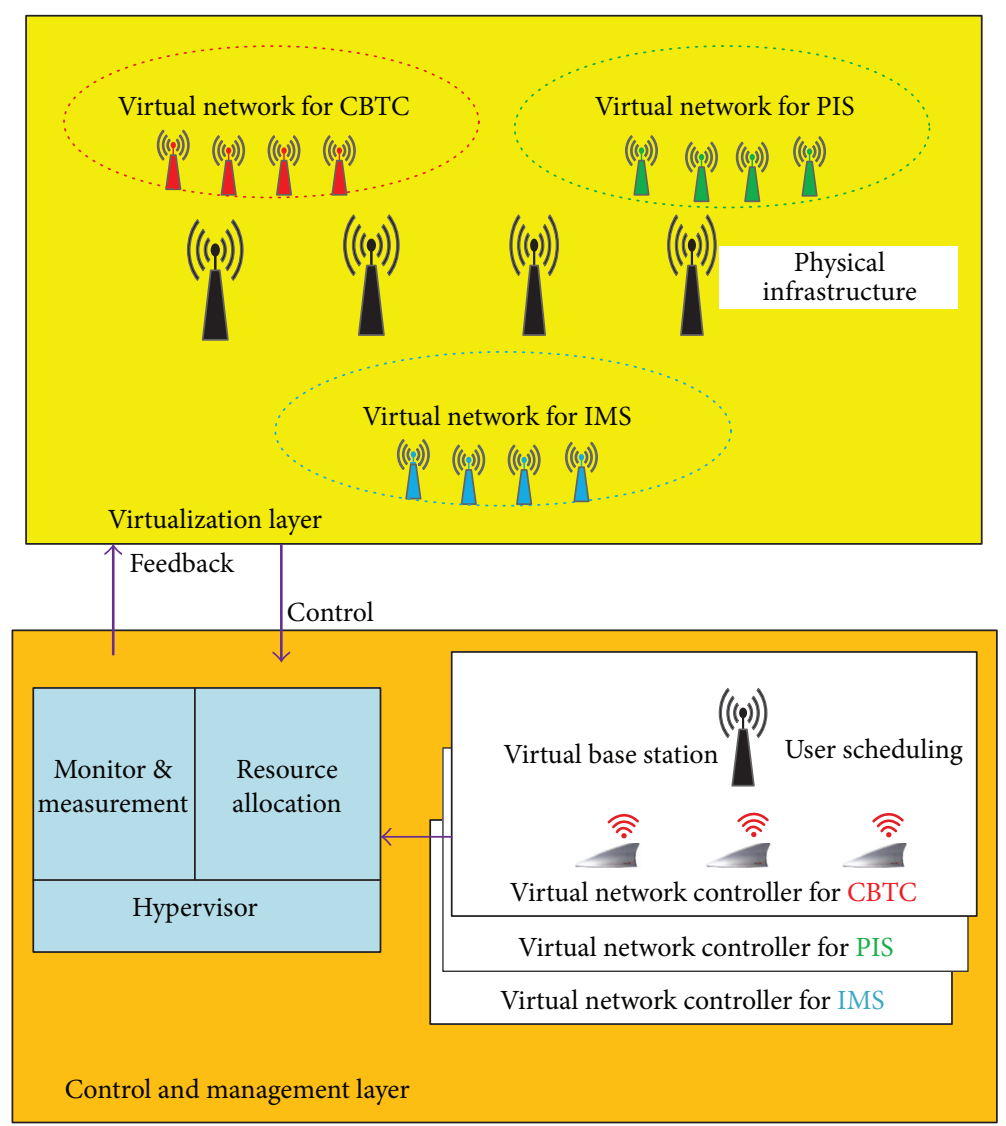

FIgURE 4: A framework of using wireless network virtualization in the proposed system.

granularity of physical wireless resources for the hypervisor. Each VBS needs a certain amount of subchannels to complete the QoS requirement for applications. We define $m_{n}$ as a subchannel of BSn. $\mathscr{M}_{n}$ is the set of all available channels of physical BSn, given the legal frequency spectrum. We assume power is evenly distributed in each channel. The hypervisor can accurately obtain the Channel state Information (CSI), available spectrum, and the QoS requirement of application stations. In order to improve the utilization of spectrum resource, each subchannel can adopt different modulation mode according to the channel state information.

The virtual resource allocation optimization can be described as maximizing the total application satisfaction on the condition of system constraints. The strictly concave, monotonically increasing, and continuously differentiable logarithmic utility function [20] is used to ensure proportionally fair resource allocation. The Opt-U1 formulations are given as follows:

\section{Opt-U1:}

$$
\begin{aligned}
\max _{\alpha, \beta} & \sum_{k \in K, v_{k} \in V_{k}} \sum_{n \in N, m_{n} \in M_{n}} \log \left(\frac{\Gamma^{P}\left(\alpha_{v_{k} n}, \beta_{v_{k} m_{n}}\right)}{\Psi^{P}}+\frac{\Gamma^{I}\left(\alpha_{v_{k} n}, \beta_{v_{k} m_{n}}\right)}{\Psi^{I}}+\frac{\Gamma^{C}\left(\alpha_{v_{k} n}, \beta_{v_{k} m_{n}}\right)}{\Psi^{C}}\right) \\
\text { s.t. } & \sum_{n \in \mathcal{N}} \alpha_{v_{k} n}=1, \quad \alpha_{v_{k} n} \in\{0,1\}, \forall k \in \mathscr{K}, \forall v_{k} \in \mathscr{V}_{k} \\
& \sum_{k \in \mathscr{K}, v_{k} \in \mathscr{V}_{k}} \beta_{v_{k} m_{n}}=1, \quad \beta_{v_{k} m_{n}} \in\{0,1\}, \forall n \in \mathscr{N}, \forall m_{n} \in \mathscr{M}_{n} \\
& \tau_{v_{k} \mathrm{CBTC}}^{\mathrm{CBTC}} \leq T_{\text {req, }}
\end{aligned}
$$

where $\Gamma^{P}(x), \Gamma^{I}(x)$, and $\Gamma^{C}(x)$ are the reward function for applications with virtual resource association strategies $\alpha_{v_{k} n}$ and $\beta_{v_{k} m_{n}}$ and $\Psi^{P}, \Psi^{I}$, and $\Psi^{C}$ denote the suggested performance value for different applications. $\alpha_{v_{k} n}$ and $\beta_{v_{k} m_{n}}$ 
are assignment indicators. If application station $v_{k}$ is assigned to BS $n$ and subchannel $m$ is assigned to user $v_{k}, \alpha_{v_{k} n}=$ 1 , and $\beta_{v_{k} m_{n}}=1$; otherwise $\alpha_{v_{k} n}=0$, and $\beta_{v_{k} m_{n}}=0$. An application station is only served by one BS, and one subchannel is not assigned to multiple application stations. The inequality reflects the fact that the transmission delay $\tau_{v_{k}}$ of CBTC application station cannot exceed its requirement threshold $T_{\text {req }}$.

When we define QoS satisfaction level (QoSL) of application station $\mathrm{SL}_{v_{k}}$ as

$$
\mathrm{SL}_{v_{k}}=\frac{\sum_{n \in N, m_{n} \in M_{n}} \Gamma^{X}\left(\alpha_{v_{k} n}, \beta_{v_{k} m_{n}}\right)}{\Psi^{X}}, \quad X=P, I, C,
$$

the optimization functions can be transformed as follows:

$$
\text { Opt-U2: }
$$

$$
\max _{\alpha, \beta} \sum_{k \in \mathscr{K}, v_{k} \in \mathscr{V}_{k}} \log \left(\mathrm{SL}_{v_{k}}\right) .
$$

For the PIS and CCTV application, our objective is to maximize their data transmission throughput and minimize their jitter delay. Therefore, the reward function for these two applications can be defined as

$$
\Gamma_{P}\left(\alpha_{v_{k} n}, \beta_{v_{k} m_{n}}\right)=\Gamma_{I}\left(\alpha_{v_{k} n}, \beta_{v_{k} m_{n}}\right)=\alpha_{v_{k} n} \beta_{v_{k} m_{n}} \Re_{v_{k} m_{n}}
$$

or

$$
\Gamma_{P}\left(\alpha_{v_{k} n}, \beta_{v_{k} m_{n}}\right)=\Gamma_{I}\left(\alpha_{v_{k} n}, \beta_{v_{k} m_{n}}\right)=\alpha_{v_{k} n} \beta_{v_{k} m_{n}} \Xi_{v_{k} m_{n}},
$$

where $\Re_{v_{k} m_{n}}$ is the achievable data rate between the subchannel $m_{n}$ and user $v_{k}$. It is a function of the subchannel available bandwidth $B_{m_{n}}$, the SNR $\xi$, and the bit error rate BER and can be computed as follows [21]:

$$
\mathfrak{R}_{v_{k} m_{n}}=B_{m_{n}} * \log _{2}\left(1+\frac{-1.5 * \xi}{\ln \left(5 * P_{v_{k}}^{\mathrm{BER}}\right)}\right) .
$$

$\Xi_{v_{k} m_{n}}$ is the jitter delay when subchannel $m_{n}$ is used for user $v_{k}$.

For the CBTC application, it is important to maintain the quick response time between the train and ground. Therefore, the reward function that reflects the transmission delay can be defined as

$$
\Gamma_{C}\left(\alpha_{v_{k} n}, \beta_{v_{k} m_{n}}\right)=\alpha_{v_{k} n} \beta_{v_{k} m_{n}} \Upsilon_{v_{k} m_{n}},
$$

where $\Upsilon_{v_{k} m_{n}}$ is the achievable data transmission delay.

Combined with small-scale fading and large-scale fading, we get the received SNR $\xi$ as

$$
\xi=P_{t}-P_{\text {loss }}+\chi+10 \log _{10}(\vartheta)+G_{t}+G_{r}-P_{\text {noise }},
$$

where $P_{t}$ is the transmitted power, $P_{\text {loss }}$ is the large-scale path loss, $\vartheta$ is a Rayleigh random variable with a mean of 1 when we use Rayleigh distribution to describe the fading envelope, $\chi$ is a Gaussian random variable with a variance of $\varsigma$ and a mean of $0, G_{t}$ and $G_{r}$ are the antenna gains for the transmitter and receiver, respectively, and $P_{\text {noise }}$ is the noise power. The path loss value $P_{\text {loss }}$ is dependent on the working frequency and transmission environment. In this paper, we use the path loss model described in [21].

The BER is determined by the suggested packet loss rate PLR given in Table 1. This is because, given the link BER, the Frame Error Rate FER and PLR are computed as

$$
\begin{aligned}
& \text { FER }=1-(1-\mathrm{BER})^{L}, \\
& \mathrm{PLR}=\mathrm{FER}^{\mathrm{MR}},
\end{aligned}
$$

where $L$ is the packet length and $M R$ is the maximum transmission time.

In this paper, we take LTE link layer as an example to compute the end to end transmission delay. LTE is a new generation of wireless communication technology, and it has become the dominant train ground communication technology for next-generation CBTC systems [15]. In LTE systems, Hybrid Automatic Repeat Quest (HARQ) is used as an error control code. Given a retransmission time $R$, the transmission delay can be computed as

$$
\Upsilon_{v_{k} m_{n}}(R)=T_{\text {data }}+R \times T_{\mathrm{RTT}},
$$

where $T_{\text {data }}$ is the packet transmission time dependent on transmission rate.

$T_{\mathrm{RTT}}$ is the Round Trip Time (RTT), which is approximately computed as

$$
T_{\mathrm{RTT}}=T_{\mathrm{data}}^{u}+T_{\mathrm{data}}^{d}+T_{\text {process }},
$$

where $T_{\text {data }}^{u}$ and $T_{\text {data }}^{d}$ are the uplink and downlink data transmission delay, and $T_{\text {process }}$ is the process time at BS and application stations.

Given the retransmission time with $R$ times retransmission, the average transmission time with maximum retransmission time MR can be computed as

$$
\begin{aligned}
T_{\mathrm{MR}}^{\mathrm{ave}}= & (1-\mathrm{FER}) * \Upsilon_{v_{k} m_{n}}(0)+\mathrm{FER}(1-\mathrm{FER}) \\
& * \Upsilon_{v_{k} m_{n}}(1)+\cdots+\mathrm{FER}^{\mathrm{MR}-1}(1-\mathrm{FER}) \\
& * \Upsilon_{v_{k} m_{n}}(\mathrm{MR}-1) .
\end{aligned}
$$

The jitter delay is considered as the standard deviation of transmission delay at any slot. Therefore, with the maximum retransmission time $\mathrm{MR}$, the jitter delay can be computed as

$$
\begin{aligned}
& \mathrm{JD}_{\mathrm{MR}} \\
& =\sqrt{\left(\Upsilon_{v_{k} m_{n}}(0)-T_{\mathrm{MR}}^{\mathrm{ave}}\right)^{2}+\cdots+\left(\Upsilon_{v_{k} m_{n}}(\mathrm{MR})-T_{\mathrm{MR}}^{\mathrm{ave}}\right)^{2}} .
\end{aligned}
$$

\section{Problem Transformation}

It is hard to solve problem Opt-U1 based on the following reasons. First, too many constraints make the problem becomes complex. And next, due to the Boolean value of $\left\{\alpha_{v_{k} n}\right\}$ and $\left\{\beta_{v_{k} m_{n}}\right\}$, both the objective function and the feasible set of Opt-U1 are not convex. 
According to the method in [22], the binary variables of $\left\{\alpha_{v_{k} n}\right\}$ and $\left\{\beta_{v_{k} m_{n}}\right\}$ can be relaxed (i.e., we assume that $0 \leq$ $\alpha_{v_{k} n} \leq 1$ and $0 \leq \beta_{v_{k} m_{n}} \leq 1$, for all $\left.k, n\right)$. We define $\omega_{v_{k} n}=$ $\alpha_{v_{k} n} \beta_{v_{k} n}$, and

$$
\beta_{v_{k} n}=\sum_{m_{n} \in \mathscr{M}_{n}} \beta_{v_{k} m_{n}}
$$

where $\beta_{v_{k} n} \in[0,1]$ is used to denote the proportion of wireless resource allocated by BSn to user $v_{k}$. Then the problem Opt-U2 obtains an equivalent transformation as follows:

Opt-U3:

$$
\begin{array}{ll}
\max _{\alpha, \beta} & \sum_{k \in \mathscr{K}, v_{k} \in \mathscr{V}} \sum_{k \in \mathcal{N}} \alpha_{v_{k} n} \log \left(\frac{\mathfrak{R}_{v_{k} n} \omega_{v_{k} n}}{\Psi^{P} \alpha_{v_{k} n}}\right. \\
& \left.\frac{\mathfrak{R}_{v_{k} n} \omega_{v_{k} n}}{\Psi^{I} \alpha_{v_{k} n}}+\frac{\Xi_{v_{k} n} \omega_{v_{k} n}}{\Psi^{P} \alpha_{v_{k} n}}+\frac{\Xi_{v_{k} n} \omega_{v_{k} n}}{\Psi^{I} \alpha_{v_{k} n}}+\frac{\Upsilon_{v_{k} n} \omega_{v_{k} n}}{\Psi^{C} \alpha_{v_{k} n}}\right) \\
\text { s.t. } \quad \sum_{n \in \mathcal{N}} \alpha_{v_{k} n}=1, \quad \alpha_{v_{k} n} \in\{0,1\}, \forall k \in \mathscr{K}, \forall v_{k} \in \mathscr{V}_{k} \\
& \sum_{k \in \mathscr{K}, v_{k} \in \mathscr{V}_{k}} \omega_{v_{k} n} \leq 1, \quad \forall n \in \mathcal{N} .
\end{array}
$$

Obviously, when $\alpha_{v_{k} n}=0$, we have $\beta_{v_{k} n}=0$, which means that the application station is not associated with any BS. Literature [23] gives the proof of the convexity for problem (14).

\section{Virtual Resource Allocation Using ADMM}

As a general solution, the CVX tool can be used to solve the convex program in (14). Given the optimal association indicator matrix $X^{*}=\left\{\alpha_{v_{k} n}\right\}$ and the optimal resource allocation indicators matrix $Y^{*}=\left\{\beta_{v_{k} n}\right\}$ at time $t$, the corresponding allocation scheme can be described as

$$
\begin{aligned}
& X^{*}=\arg \max _{\alpha} \sum_{k \in K, v_{k} \in V_{k}} \sum_{n \in N} \frac{\partial U_{v_{k}}\left(\mathrm{SL}_{v_{k}}\right)}{\mathrm{SL}_{v_{k}}} \\
& \cdot\left(\frac{\Re_{v_{k} n} \beta_{v_{k} n}}{\Psi^{P}}+\frac{\Re_{v_{k} n} \beta_{v_{k} n}}{\Psi^{I}}\right. \\
& \left.+\frac{\Xi_{v_{k} n} \beta_{v_{k} n}}{\Psi^{P}}+\frac{\Xi_{v_{k} n} \beta_{v_{k} n}}{\Psi^{I}}+\frac{\Upsilon_{v_{k} n} \beta_{v_{k} n}}{\Psi^{C}}\right) \\
& Y^{*}=\arg \max _{\beta} \sum_{k \in K, v_{k} \in V_{k} n \in N} \frac{\partial U_{v_{k}}\left(\mathrm{SL}_{v_{k}}\right)}{\mathrm{SL}_{v_{k}}} \\
& \cdot\left(\frac{\Re_{v_{k} n} \alpha_{v_{k} n}}{\Psi^{P}}+\frac{\Re_{v_{k} n} \alpha_{v_{k} n}}{\Psi^{I}}+\frac{\Xi_{v_{k} n} \beta_{v_{k} n}}{\Psi^{P}}+\frac{\Xi_{v_{k} n} \beta_{v_{k} n}}{\Psi^{I}}\right. \\
& \left.+\frac{\Upsilon_{v_{k} n} \beta_{v_{k} n}}{\Psi^{C}}\right) \cdot
\end{aligned}
$$

Observed from the above two formulas, to get the optimal allocation scheme, the centralized algorithm must obtain the achievable rate $\Re_{v_{t} n}$ of all users at time $t$ and the average satisfaction level $\partial U_{v_{k}}\left(\mathrm{SL}_{v_{k}}\right) / \mathrm{SL}_{v_{k}}$ of all users at time $t-1$. This results in a relatively large amount of calculation for the high speed urban rail transit system. In order to overcome it, we use ADMM to solve the convex problem. ADMM is a computing framework for optimization. It is suitable for solving distributed convex optimization problem, especially the statistical learning problems [18].

In order to use ADMM to solve the convex optimization, local copies of the global assignment indicators are introduced. Roughly speaking, each local variable can be interpreted as the information owned by each BS about the corresponding global assignment indicators variable.

To drive the local copies into consensus, we use distributed consensus ADMM method [18]. Let $\Delta=$ $\left\{\alpha_{v_{k} n}, \forall v, k, n\right\}$ denote the vector of assignment indicators and $\varepsilon_{n}$ denote the local copy of $\Delta$ at BSn. To the consensus constraints, we introduce an auxiliary variable $\varepsilon_{v_{k} n}$ which represents the local copies of our assignment indicators as equality constraints:

$$
\varepsilon_{v_{k} n}=\alpha_{v_{k} n}, \quad \forall v, k, n
$$

Given the local vectors $\Omega_{n}=\left\{\varepsilon_{n}, \forall n\right\}$ and $\ell_{n}=\left\{\omega_{n}, \forall n\right\}$, we define a feasible local variable set for each BSn $\in \mathscr{N}$. The constraints in (15b) can be decomposed into $N$ independent convex sets as

$$
\Lambda_{n}=\left\{\Omega_{n}, \ell_{n} \mid \sum_{k \in \mathscr{K}, v_{k} \in \mathscr{V}_{k}} \omega_{v_{k} n} \leq 1, \forall n \in \mathcal{N}\right\}
$$

and an associated local utility function as

$$
\begin{aligned}
& u_{n} \\
& = \begin{cases}-\sum_{k \in \mathscr{K}, v_{k} \in \mathscr{V}_{k}} \varepsilon_{v_{k} n} \log \left(\frac{\Re_{v_{k} n} \omega_{v_{k} n}}{\gamma_{v_{k}}^{\mathrm{req}} \varepsilon_{v_{k} n}}\right) \Omega_{n}, & \ell_{n} \in \Lambda_{n} \\
\infty & \text { otherwise. }\end{cases}
\end{aligned}
$$

Using (17) and (18) and the auxiliary variable $\varepsilon_{v_{k} n}$, we can compactly write the global consensus problem (14) as

$$
\begin{array}{ll}
\min & \sum_{n \in \mathcal{N}} u_{n}\left(\Omega_{n}, \ell_{n}\right) \\
\text { s.t. } & \varepsilon_{v_{k} n}=\alpha_{k_{i} n}, \quad \forall v, k, n .
\end{array}
$$

Then the augmented Lagrangian function for (19) can be rewritten as

$$
\begin{aligned}
\mathscr{L}_{\rho}\left(\left\{\Omega_{n}, \ell_{n}\right\},\{\Delta\},\left\{\sigma_{n}\right\}\right) \\
=\sum_{n \in \mathcal{N}} u_{n}\left(\Omega_{n}, \ell_{n}\right)+\sum_{n \in \mathcal{N}} \sum_{k \in \mathscr{K}, v_{k} \in \mathscr{V}_{k}} \sigma_{v_{k} n}\left(\varepsilon_{v_{k} n}-\alpha_{v_{k} n}\right) \\
\quad+\left(\frac{\rho}{2}\right) \sum_{n \in \mathcal{N}} \sum_{k \in \mathscr{K}, v_{k} \in \mathscr{V}_{k}}\left(\varepsilon_{v_{k} n}-\alpha_{v_{k} n}\right)^{2},
\end{aligned}
$$


where $\sigma_{v_{k} n}$ is the Lagrange multipliers related to the constraints of consensus in problem (19) and $\rho>0$ is a penalty parameter for adjusting the convergence speed of the ADMM [18].

The basic idea of ADMM is that convex optimization is broken into smaller partitions, each of which are then easier to handle. The ADMM method is composed of successive optimization steps by updating the primal and dual variables alternately. For optimization, at iteration $q$ we need to take the following steps:

$$
\begin{gathered}
\left\{\Omega_{n}, \ell_{n}\right\}_{n \in \mathscr{N}}^{q+1}=\arg \min \left\{u_{n}\left(\Omega_{n}, \ell_{n}\right)+\sum_{k \in \mathscr{K}, v_{k} \in \mathscr{V}_{k}} \sigma_{v_{k} n}{ }^{q}\left(\varepsilon_{v_{k} n}-\alpha_{v_{k} n}{ }^{q}\right)+\left(\frac{\rho}{2}\right) \sum_{k \in \mathscr{K}, v_{k} \in \mathscr{V}_{k}}\left(\varepsilon_{v_{k} n}-\alpha_{v_{k} n}{ }^{q}\right)^{2}\right\} \\
\{\Delta\}^{q+1}=\arg \min \left\{\sum_{n \in \mathscr{N}} \sum_{k \in \mathscr{K}, v_{k} \in \mathscr{V}_{k}} \sigma_{v_{k} n}{ }^{q}\left(\varepsilon_{v_{k} n}{ }^{q+1}-\alpha_{v_{k} n}\right)+\left(\frac{\rho}{2}\right) \sum_{n \in \mathscr{N}} \sum_{k \in \mathscr{K}, v_{k} \in \mathscr{V}_{k}}\left(\varepsilon_{v_{k} n}{ }^{q+1}-\alpha_{v_{k} n}\right)^{2}\right\} \\
\left\{\sigma_{n}\right\}_{n \in \mathcal{N}}^{q+1}=\sigma_{n}{ }^{q}+\rho\left(\Omega_{n}{ }^{q+1}-\Delta^{q+1}\right)
\end{gathered}
$$

\section{Simulation Results and Discussions}

In this section, we use MATLAB 2015b to carry out simulation. Simulation results are presented to illustrate the optimal performance of the proposed algorithm.

In order to simplify the simulation model, we consider that there are four physical base stations in the integrated train ground communication system and each physical BS can be virtualized into three virtual base stations, providing three services, CBTC, PIS, and CCTV, respectively, as shown in Figure 5. Among them, the red network base stations BS1 and BS2 belong to the infrastructure InP1, and the blue network base stations BS3 and BS4 belong to the infrastructure InP2. BS1 and BS3 or BS2 and BS4 cover the same geographic area, which forms the redundant coverage and ensures the reliability of CBTC systems. We assume wireless virtualization can be used between different InPs. Wireless spectrum resources can be shared by multiple virtual base stations virtualized from BS1 and BS3 or BS2 and BS4. For application stations, there is no obvious difference between different infrastructures as if all resources are within the same resource pool (e.g., $f_{\mathrm{CBTC}}, f_{\mathrm{PIS}}$, and $f_{\mathrm{CCTV}}$ are within the same resource pool).

In order to illustrate the performance improvement of our proposed algorithm, we compare it with the existing algorithm. In the existing algorithm, the application stations connect base stations providing the maximum received signal strength (RSS), and each BS carries out wireless spectrum resource allocation with proportional fairness. We name the existing scheme as Max-RSS.

As we can observe from Figure 6(a), under the Max-RSS scheme, some of the application station satisfaction level is less than zero, which makes the QoS of these application stations not guaranteed. However, the QoS requirement of all application stations can be satisfied with the proposed WVRA scheme as shown in Figure 6(b). This is because there are more than one application station associated with the same base station at the same time, but the application stations connect base stations providing the maximum received signal strength (RSS) when Max-RSS scheme is adopted, and the QoS guarantee is not considered. On the contrary, the WVRA scheme fully considers the QoS guarantee, base station (BS) load balance, and application station fairness. By taking this scheme, the QoS requirement of each application station is guaranteed.

Next, we assess the fairness performance of different algorithms using fairness index described in literature [24]. If the fairness index is close to 1, it means the algorithm has a higher degree of fairness, and vice versa. The fairness index is defined as follows:

$$
\mathrm{FI}\left(\mathrm{SL}_{v_{k}}\right)=\frac{\left(\sum_{k=1}^{k=K} \mathrm{SL}_{v_{k}}\right)^{2}}{K \sum_{k=1}^{k=K} \mathrm{SL}_{v_{k}}^{2}} .
$$

As we can observe from Figure 7, with the gradual increase of the application stations in the cell, the Max-RSS algorithm cannot guarantee the fair distribution of virtual resources. It is mainly because the wireless resources are limited, and strong competition between applications leads to the decrease of fairness. However, our proposed algorithm WVRA effectively ensures the fairness of the virtual resource allocation. Although application stations continue to increase, the fairness index keeps unchanged, which means the virtual resources can still be fairly allocated.

In order to verify the jitter delay performance improvement of PIS and CCTV applications, we illustrate the transmission delay of CCTV application in Figure 8. The transmission delays of our proposed WVRA scheme are more volatile compared with the existing scheme, which means the proposed WVRA scheme performs better in terms of jitter delay. This is due to the fact that the WVRA scheme fully considers the QoS requirement of all applications, and one of the direct optimization objectives is to minimize the jitter delay of PIS and CCTV. We also notice that WVRA scheme sacrifices part of transmission delay performance to realize its optimization objective.

We study the spectrum allocation between virtual base station of physical base stations and compare each BS load fluctuation in Figures 9 and 10, respectively. As illustrated in Figure 9, the spectrum allocated to VBS1 with the CBTC application is approximately unchanged in each time slot. 


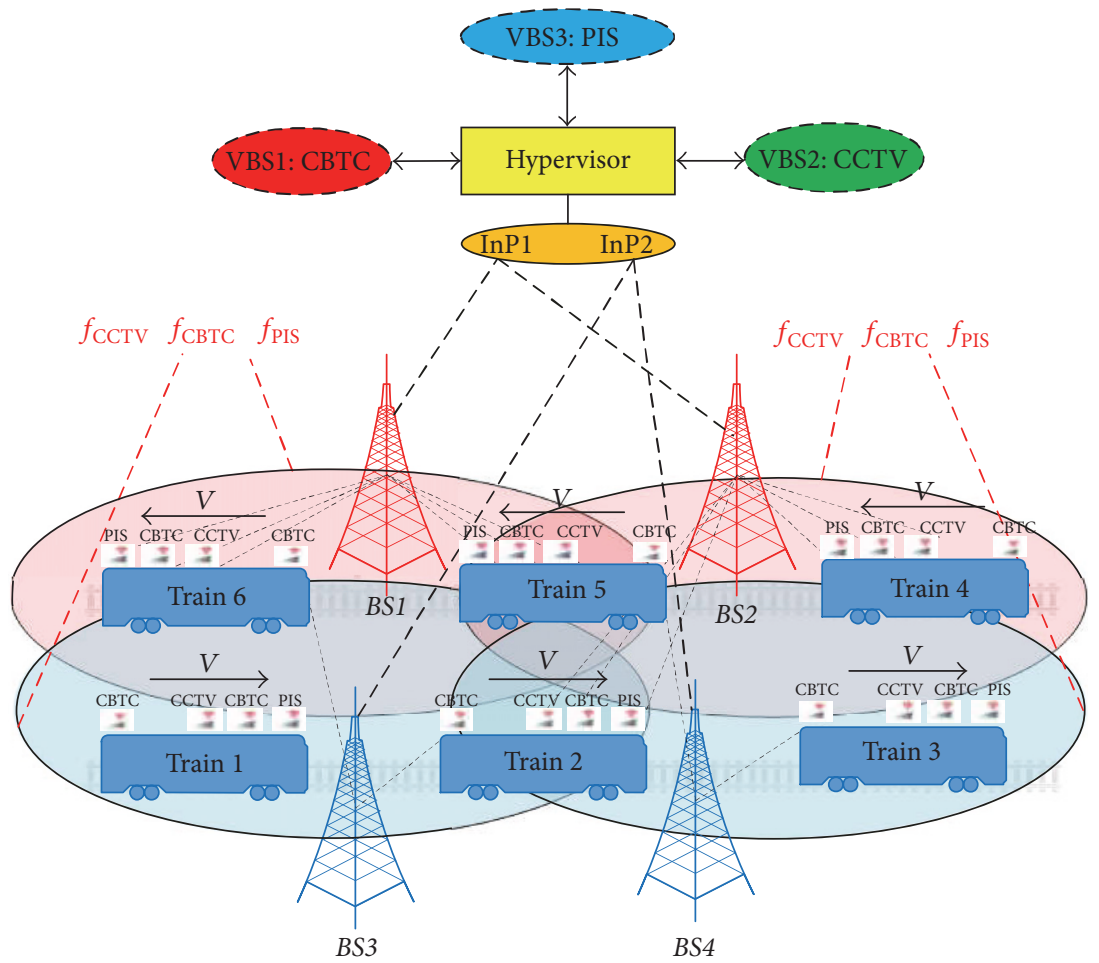

FIGURE 5: System model with four base stations and three virtual base stations for CBTC, PIS, and CCTV, respectively.

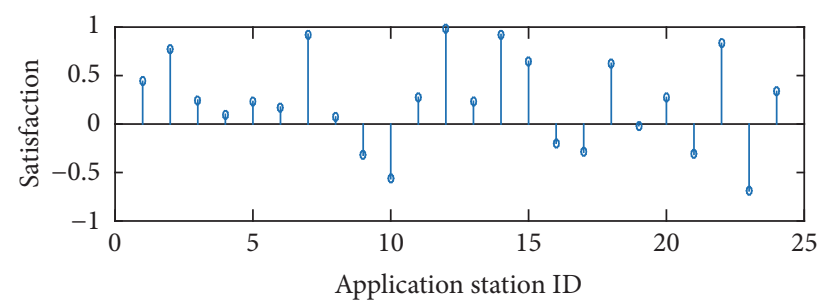

(a) Max-RSS

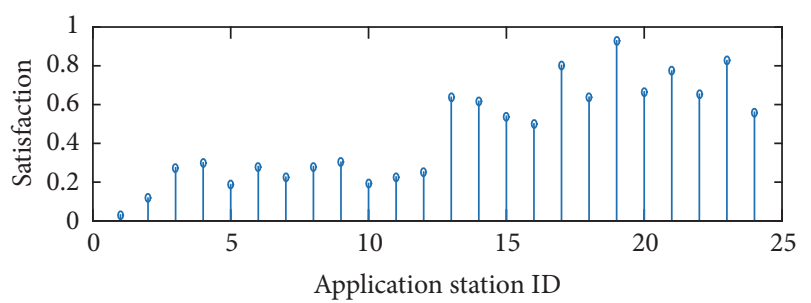

(b) WVRA

FIGURE 6: QoS satisfaction level improvement under different algorithms.

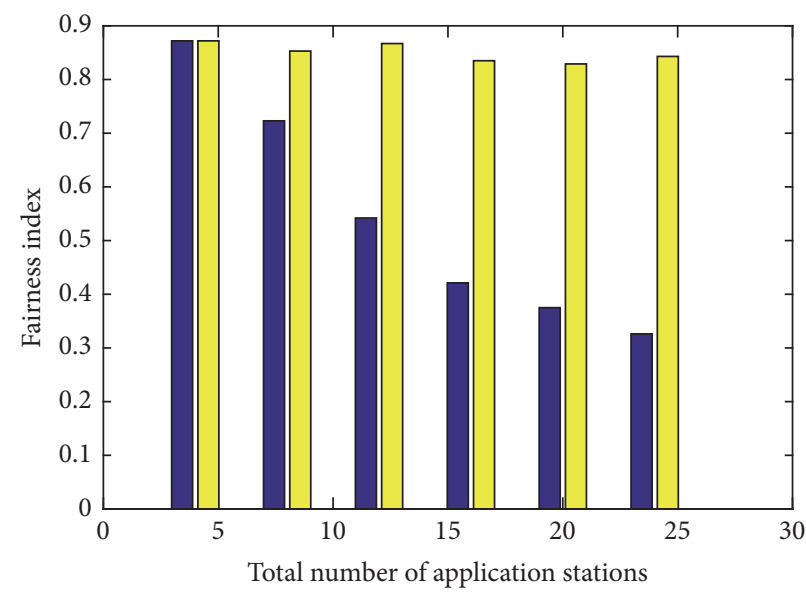

Max-RSS

WVRA

FIGURE 7: Comparison of fairness index for different resource allocation algorithms. 


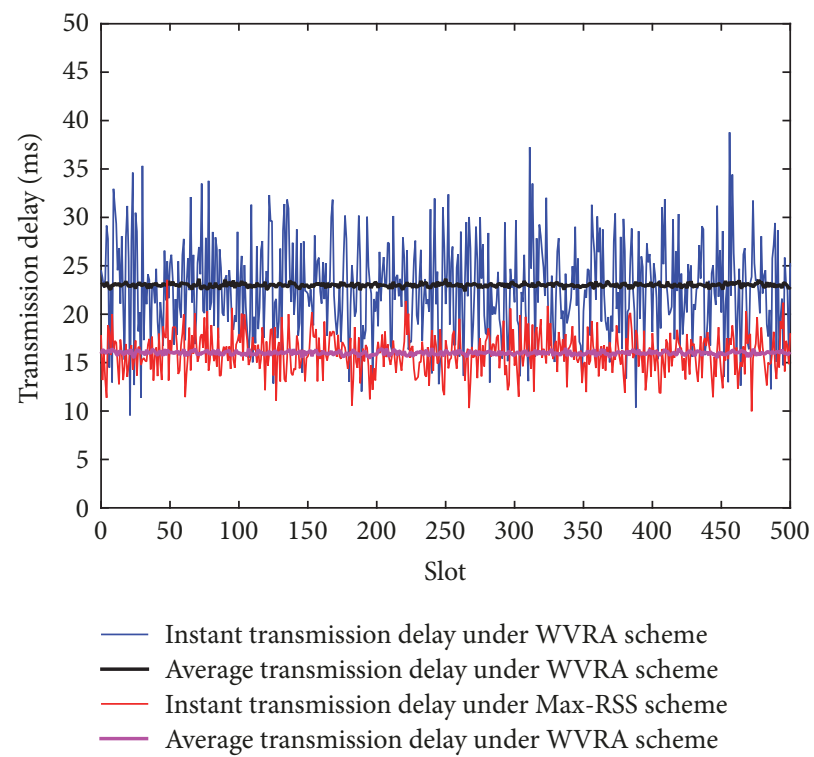

Figure 8: Transmission delay of the CCTV application under different scheme.

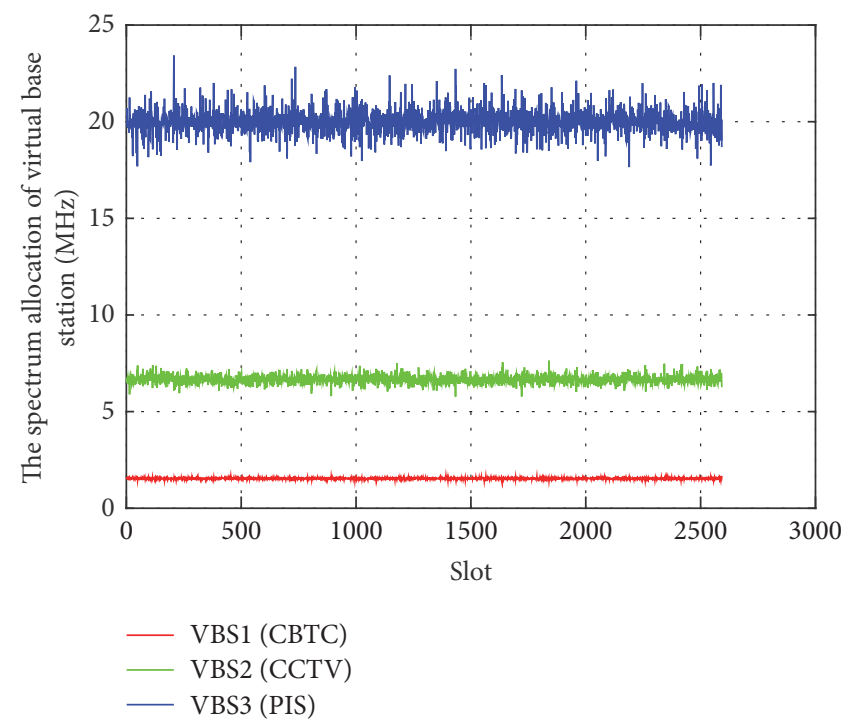

FIGURE 9: Wireless spectrum allocation for each VBS using WVRA.

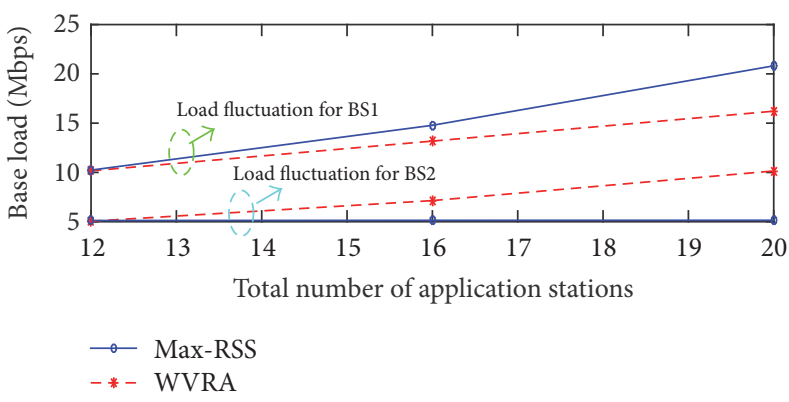

(a) BS1 and BS2 load fluctuation

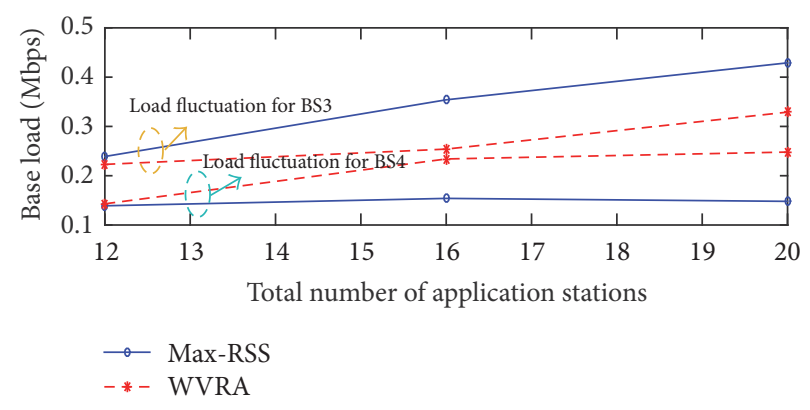

(b) BS3 and BS4 load fluctuation

FIGURE 10: BS load using different resource allocation algorithms. 
This is because the optimization objective of CBTC application is not the transmission data rate, and the required spectrum is relatively stable. As for the other two VBSs that carry the PIS and CCTV application traffic, we can notice that the spectrum allocated to them changes at each time slot with the traffic load. This is due to the fact that maximizing the transmission throughput needs large amount of spectrums.

In order to verify the load balance performance, we set up the simulation environment where the BS1 and BS3 give a higher received signal strength in the overlap zone. Figure 10 shows the change of base station load when the number of application stations increases in its coverage area. The red line in the figure represents the effect of the Max-RSS scheme on the base station load. The blue line represents the effect of the WVRA scheme on the base station load. The green oval represents BS1 load fluctuations. The aquamarine blue oval represents BS2 load fluctuations. The yellow oval represents BS3 load fluctuation. The final oval represents the BS4 load fluctuations. As shown in Figure 10, we can observe that, by using the Max-RSS scheme, the loads of BS1 and BS3 increase constantly, while the loads of BS2 and BS4 do not change with the increase of the number of the application stations. This is due to the fact that application station fairness is not considered under this scheme. On the contrary, the WVRA scheme successfully separates part of the load of BS1 and BS3 to the more lightly loaded BS2 and BS4, although BS2 and BS4 offer a lower instantaneous received signal strength than BS1 and BS3.

\section{Conclusions}

In this paper, we have proposed a framework of using network virtualization in an integrated train ground communication system. We have formulated and transformed the QoS-aware virtual resource allocation problem in the integrated system to a convex optimization problem. We define the QoS salification level parameter to reflect the application satisfaction. The final objective is fairness driven optimization function based on QoS guarantee, base station load balance, and application station fairness. We use the distributed method based on ADMM to solve the convex problem. Simulation results indicate that our algorithm can guarantee the QoS requirement of all application stations. Meanwhile, the traffic load of different base stations can be balanced to achieve better performance of the whole system.

\section{Simulation Parameters}

$\begin{array}{ll}\tau: & \text { Slot time, } 50 \mathrm{~ms} \\ P_{t}: & \text { Transmission power, } 43 \mathrm{dBm} \\ G_{t}, G_{r}: & \text { Antenna gain, } 8 \mathrm{~dB} \\ L: & \text { Average packet size, } 200 \text { bytes } \\ \mathrm{MR}: & \text { Maximum transmission time, } 6 \\ T_{\text {process }}: & \text { Process time at BS or station, } 10 \mathrm{~ms} \\ D: & \text { Average base station space, } 1000 \mathrm{~m} \\ v: & \text { Train speed, } 80 \mathrm{~km} / \mathrm{h} \\ \varsigma: & \text { Shadowing fading standard deviation, } 8 \\ P_{\text {noise }}: & \text { Noise power, }-100 \mathrm{dbm} .\end{array}$

\section{Conflicts of Interest}

The authors declare that there are no conflicts of interest regarding the publication of this paper.

\section{Acknowledgments}

This paper was supported by grants from Beijing Natural Science Foundation (L171004, I18E300010), by the National Natural Science Foundation of China (no. 61603026), by Technological Research and Development Program of China Railway Corporation (Grant 2016X008-B), by Fundamental Research Funds for the Central Universities (2015JBM005), by Projects RCS2017ZT003, RCS2017ZT006, and KIE017001531, and by the Beijing Key Laboratory of Urban Rail Transit Automation and Control.

\section{References}

[1] T. Xu, T. Tang, C. Gao, and B. Cai, "Dependability analysis of the data communication system in train control system," Science China Technological Sciences, vol. 52, no. 9, pp. 2605-2618, 2009.

[2] R. Pascoe and T. Eichorn, "What is communication-based train control?” IEEE Vehicular Technology Magazine, vol. 4, no. 4, pp. 16-21, 2009.

[3] L. Zhu, F. R. Yu, T. Tang, and B. Ning, "Handoff Performance Improvements in an Integrated Train-Ground Communication System Based on Wireless Network Virtualization," IEEE Transactions on Intelligent Transportation Systems, vol. 18, no. 5, pp. 1165-1178, 2017.

[4] J. Moreno, J. Riera, L. De Haro, and C. Rodriguez, "A survey on future railway radio communications services: challenges and opportunities," IEEE Communications Magazine, vol. 53, no. 10, pp. 62-68, 2015.

[5] J. Farooq and J. Soler, "Radio Communication for Communications-Based Train Control (CBTC): A Tutorial and Survey," IEEE Communications Surveys \& Tutorials, vol. 19, no. 3, pp. 1377-1402, 2017.

[6] H. Jiang, V. C. M. Leung, C. Gao, and T. Tang, "MIMOassisted handoff scheme for communication-based train control systems," IEEE Transactions on Vehicular Technology, vol. 64, no. 4, pp. 1578-1590, 2015.

[7] X. U. Tian-Hua, L. I. Shu, and T. Tao, "Dependability analysis of data communication subsystem in train control system," Journal of Beijing Jiaotong University, vol. 31, no. 5, pp. 23-21, 2007.

[8] K. Guan, Z. Zhong, B. Ai, and C. Briso-Rodríguez, "Measurement and modeling of subway near shadowing phenomenon," in Proceedings of the 5th International ICST Conference on Communications and Networking in China, ChinaCom 2010, August 2010.

[9] K. Guan, Z. D. Zhong, J. I. Alonso, and C. Briso-Rodríguez, "Measurement of distributed antenna systems at $2.4 \mathrm{GHz}$ in a realistic subway tunnel environment," IEEE Transactions on Vehicular Technology, vol. 61, no. 2, pp. 834-837, 2012.

[10] A. Amanna, M. Gadniok, M. Price, J. Reed, W. Siriwongpairat, and T. Himsoon, "Railway cognitive radio: Future wireless communication systems for railways," IEEE Vehicular Technology Magazine, vol. 5, no. 3, pp. 82-89, 2010.

[11] O. B. Karimi, J. Liu, and C. Wang, "Seamless wireless connectivity for multimedia services in high speed trains," IEEE Journal 
on Selected Areas in Communications, vol. 30, no. 4, pp. 729-739, 2012.

[12] L. Tian, J. Li, Y. Huang, J. Shi, and J. Zhou, "Seamless duallink handover scheme in broadband wireless communication systems for high-speed rail," IEEE Journal on Selected Areas in Communications, vol. 30, no. 4, pp. 708-717, 2012.

[13] L. Zhu, F. Richard Yu, T. Tang, and B. Ning, "An Integrated Train-Ground Communication System Using Wireless Network Virtualization: Security and Quality of Service Provisioning," IEEE Transactions on Vehicular Technology, vol. 65, no. 12, pp. 9607-9616, 2016.

[14] H. Zhao, L. Zhu, H. Jiang, and T. Tang, "Design and performance tests in an integrated TD-LTE based train ground communication system," in Proceedings of the 2014 17th IEEE International Conference on Intelligent Transportation Systems, ITSC 2014, pp. 747-750, China, October 2014.

[15] T. Tang, K. Dai, Y. Zhang, H. Zhao, and H. Jiang, "Field test results analysis in urban rail transit train ground communication systems of integrated service using LTE-M," in Proceedings of the 19th IEEE International Conference on Intelligent Transportation Systems, ITSC 2016, pp. 2017-2021, Brazil, November 2016.

[16] F. Wang, L. Zhu, and H. Zhao, "Virtual resource allocation in a network virtualization based integrated train ground communication system," in Proceedings of the 19th IEEE International Conference on Intelligent Transportation Systems, ITSC 2016, pp. 2012-2016, Brazil, November 2016.

[17] C. Liang and F. R. Yu, "Wireless network virtualization: a survey, some research issues and challenges," IEEE Communications Surveys \& Tutorials, vol. 17, no. 1, pp. 358-380, 2015.

[18] S. Boyd, N. Parikh, E. Chu, B. Peleato, and J. Eckstein, "Distributed optimization and statistical learning via the alternating direction method of multipliers," Foundations and Trends in Machine Learning, vol. 3, no. 1, pp. 1-122, 2010.

[19] L. Zhu, F. R. Yu, B. Ning, and T. Tang, "Cross-layer handoff design in MIMO-enabled WLANs for Communication-Based Train Control (CBTC) systems," IEEE Journal on Selected Areas in Communications, vol. 30, no. 4, pp. 719-728, 2012.

[20] H. Yaïche, R. R. Mazumdar, and C. Rosenberg, "A game theoretic framework for bandwidth allocation and pricing in broadband networks," IEEE/ACM Transactions on Networking, vol. 8, no. 5, pp. 667-678, 2000.

[21] X. Qiu and K. Chawla, "On the performance of adaptive modulation in cellular systems," IEEE Transactions on Communications, vol. 47, no. 6, pp. 884-895, 1999.

[22] Q. Ye, B. Rong, Y. Chen, M. Al-Shalash, C. Caramanis, and J. G. Andrews, "User association for load balancing in heterogeneous cellular networks," IEEE Transactions on Wireless Communications, vol. 12, no. 6, pp. 2706-2716, 2013.

[23] S. Gortzen and A. Schmeink, "Optimality of dual methods for discrete multiuser multicarrier resource allocation problems," IEEE Transactions on Wireless Communications, vol. 11, no. 10, pp. 3810-3817, 2012.

[24] R. Jain, D.-M. Chiu, and W. R. Hawe, A quantitative measure of fairness and discrimination for resource allocation in shared computer system, vol. 38, Eastern Research Laboratory, Digital Equipment Corporation, Hudson, 1984. 


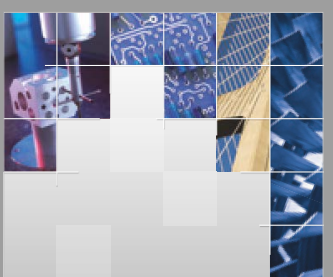

\section{Enfincering}
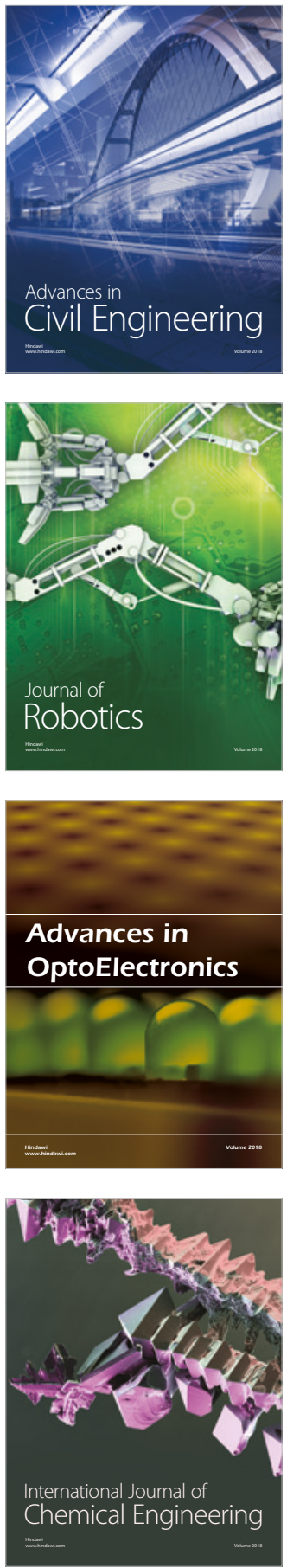

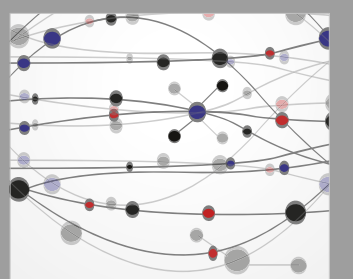

\section{Rotating \\ Machinery}

The Scientific World Journal

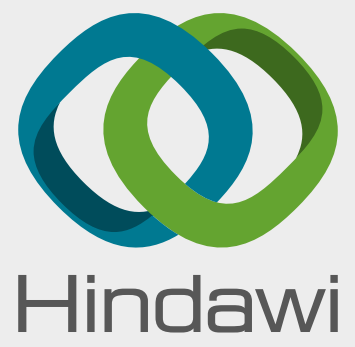

Submit your manuscripts at

www.hindawi.com
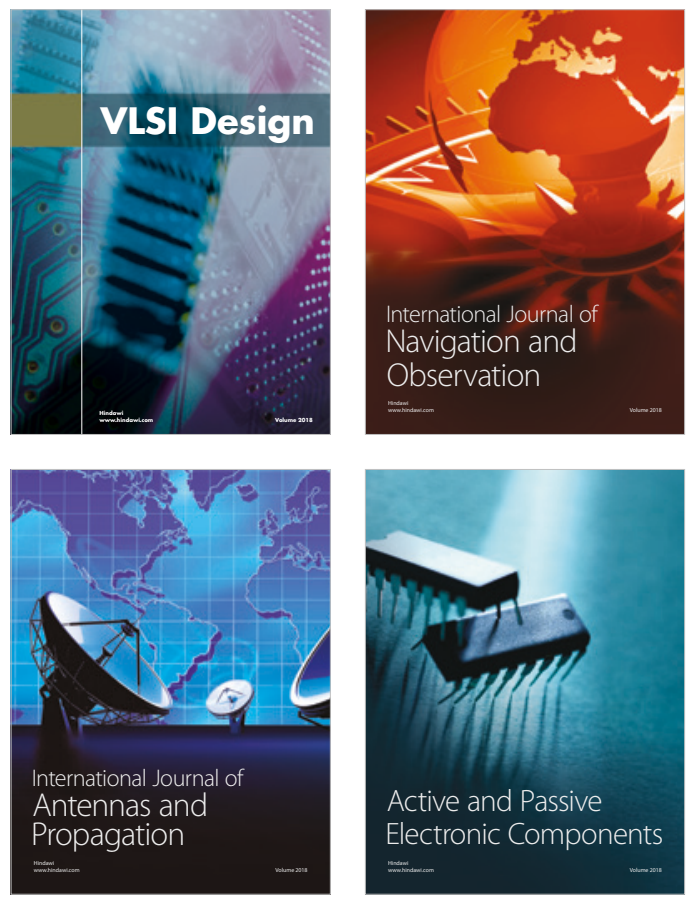
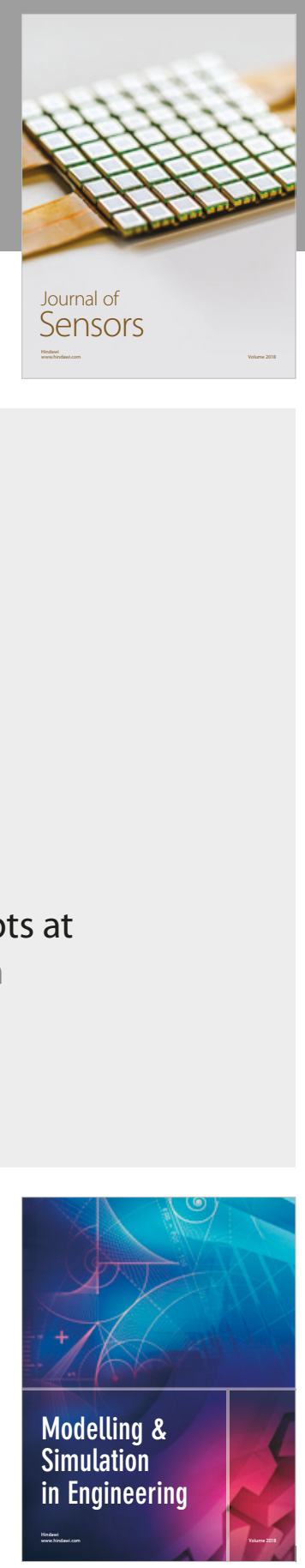

\section{Advances \\ Multimedia}
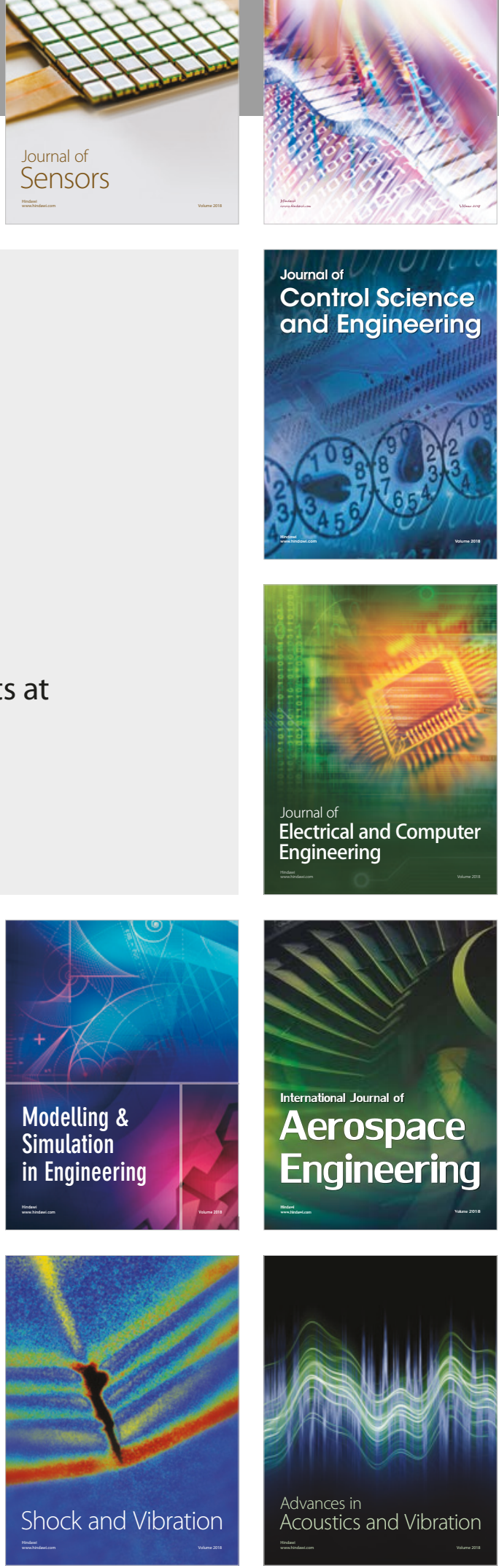